\title{
Simultaneous numerical discriminations by rats
}

\author{
HANK DAVIS and SHEREE ANNE BRADFORD \\ University of Guelph, Guelph, Ontario, Canada
}

\begin{abstract}
Four rats were trained to perform two numerical discriminations during the same experimental session. As in a previous study (Davis \& Bradford, in press), one of these tasks required the animal to enter a target tunnel solely on the basis of its ordinal position in an array of six tunnels. Of primary interest here is the fact that the subjects were simultaneously required to keep track of the cumulative number of trials during the session: After their first and second tunnel entries, subjects returned to the start box. After their third tunnel entry, subjects climbed onto a wood block in order to exit the enclosure and return to their home cages. These data constitute the first evidence that rats are capable of performing two simultaneous numerical discriminations.
\end{abstract}

Demonstrations of numerical ability in animal species have been well established (see Davis \& Memmott, 1982, for review). Many of the experiments on numerical competence in animals have involved procedures that required considerable environmental support or extensive training (e.g., Davis \& Memmott, 1983; Ferster, 1964). However, we have recently reported a procedure that yielded evidence of numerical ability in rats following relatively few training trials and under minimal deprivation conditions (Davis \& Bradford, in press). Our procedure involves teaching an animal to discriminate the ordinal position of a wooden tunnel in an array of six identical tunnels, under conditions in which all nonnumerical information (e.g., visual, temporal, spatial cues) was controlled. We speculated that the relative ease with which this discrimination was learned may have been due to the relatively natural situation under which the animals were trained: a large test area with bedding material and simulated burrows, rather than an arbitrary operant test chamber (see Menzel \& Juno, 1982, for elaboration of this position).

In the present report, we describe the use of the same technique to teach rats two simultaneous numerical discriminations. The rats were again required to leave a startbox and enter an enclosure in order to select the correct tunnel on the basis of its ordinal position. In addition, however, the rats were required to keep track of the cumulative number of trials during the session and to use the occurrence of the third trial as a cue for a unique behavioral sequence. Thus, to be successful, an animal had to return to the startbox following Trials 1 and 2 in each session, and exit the test enclosure and return to its home cage following Trial 3.

This research was supported in part by Grant A0693 from the Natural Sciences and Engineering Research Council of Canada. These data were reported at the June 1983 meeting of the Animal Behavior Society. The authors are grateful to Rachelle Perusse, Janice Steirn, and Susan Simmons for their insightful criticisms. The authors' address is: Department of Psychology, University of Guelph, Guelph, Ontario, Canada N1G 2W1.
Zorina (1982) recently examined the possibility that the sequential occurrence of trials within a session may be an effective cue for differential performance in birds. However, her results do not make clear whether this discrimination was facilitated by the detection of temporal regularities in trial length.

Although selection of the correct tunnel continued to be reinforced, as in our previous study (Davis \& Bradford, in press), our primary focus in the present experiment was on the simultaneous discrimination of trial number as the basis for successful performance. Therefore, errors consisted of premature attempts to leave the test enclosure following Trial 1 or 2, or returns to the startbox following completion of Trial 3.

\section{METHOD}

\section{Subjects}

The subjects were four 150-day-old female Long Evans rats, housed individually, with food and water freely available in their home cages. Three additional subjects were excluded after the first block of sessions for failing to reliably emit the behaviors (returning to the startbox; entering the home cage) on which the shaping of "counting trials" performance was to be based.

\section{Apparatus}

The apparatus has previously been described (Davis \& Bradford, in press). Briefly, at the start of each trial, a rat was placed in a $29 \times 17.5 \times 24.5 \mathrm{~cm}$ startbox that was attached to a $1.5 \times 0.9 \mathrm{~m}$ wooden enclosure. The enclosure was covered to an approximate depth of $3 \mathrm{~cm}$ with granulated corncob bedding material. Six wooden tunnels, each $10 \mathrm{~cm}$ wide, $22 \mathrm{~cm}$ long, and $8.9 \mathrm{~cm}$ high, were located along the left $1.5-\mathrm{m}$ wall. The front door of each tunnel was hinged at the top and swung freely, unless restricted by a $20-\mathrm{cm}$-long plywood insert that ran from the enclosure wall to the tunnel door. When the insert was in place, the door could be opened only to a depth of approximately $2 \mathrm{~cm}$, thus prohibiting access to the food bait (either a sunflower seed or a 45-mg Noyes pellet). When the insert was not in place, the animal could easily nose open the tunnel door. Although the size of the tunnel accommodated the entire animal, a typical trial consisted of a partial entry, sufficient in depth to retrieve the food bait, which was located approximately $3 \mathrm{~cm}$ inside the tunnel.

A $9 \times 9 \times 46 \mathrm{~cm}$ wood block was placed along the right $1.5-\mathrm{m}$ wall. 


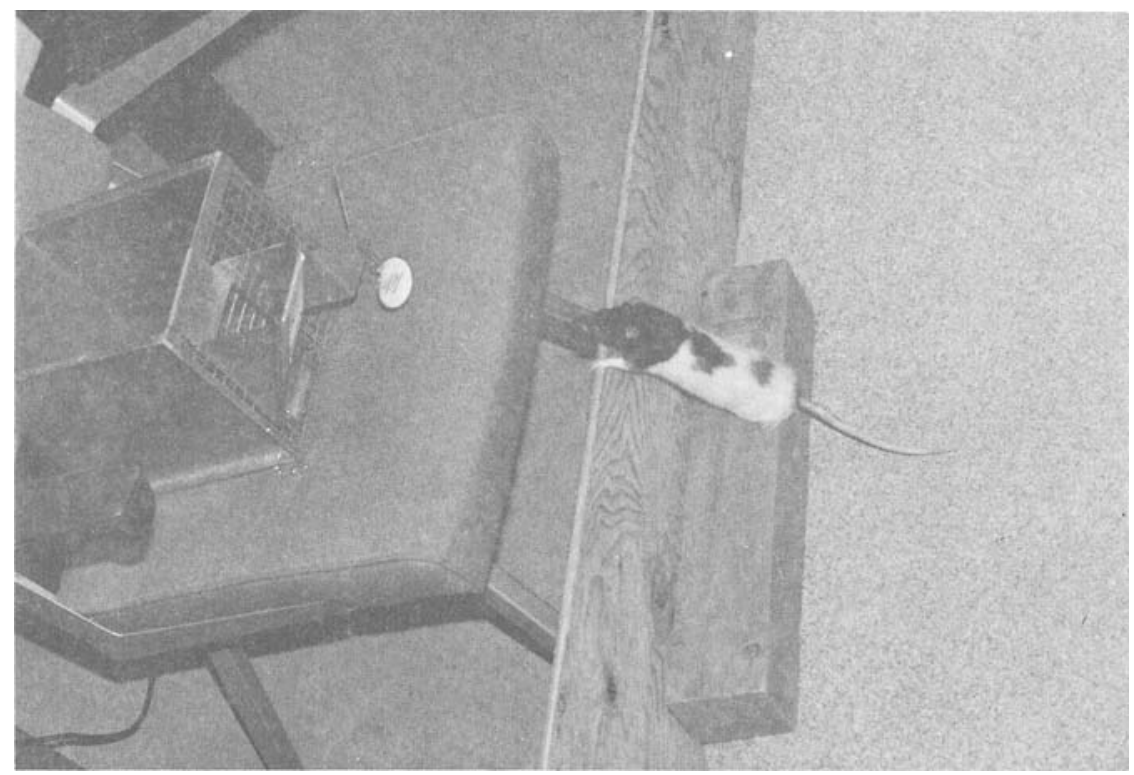

Figure 1. Illustrative view of animal climbing to top of enclosure wall in order to reach adjoining chair and home cage following Trial 3.

The test protocol also included a commercially available office chair with upholstered seat and metal arms and back, as well as the animal's own home cage, made of stainless steel mesh.

The wood block was placed in the test enclosure opposite the six tunnels and centered along the right wall. The chair was centered along the outside right wall of the enclosure, with its front legs flush to the exterior wall of the enclosure. The animal's home cage, open at the top, was placed on the seat of the chair.

\section{Procedure}

Using the method described in our previous report (Davis \& Bradford, in press), we trained each animal to enter either Tunnel 3 (Subjects $A$ and $B$ ) or Tunnel 4 (Subjects $C$ and $D$ ). At the beginning of each session, the rat was placed in the startbox. The experimenter was situated behind the startbox. When the startbox's guillotine door was lifted by the experimenter, the animal entered the enclosure, moving in a direction away from the experimenter, and chose the target tunnel by nosing open its hinged door to receive a food reward.

The training procedure for tunnel entries involved a sequence in which nonnumerical cues (e.g., olfactory or spatial) were gradually faded until performance was guided solely by the tunnel's ordinal position. The tunnel located closest to the startbox was considered to be Tunnel 1 . During the first 2 days of training, the six tunnels were equally spaced along the $1.5-\mathrm{m}$ wall. The door was removed from the target tunnel and food bait (either a sunflower seed or a $45-\mathrm{mg}$ Noyes pellet) was placed inside. Entry into the remaining five tunnels, which were not baited, was restricted by the use of wooden inserts (see description in Apparatus). After approximately 10 sessions, all tunnels were baited with food. Within the next 5 sessions, the door to the target tunnel was replaced, thus requiring the animal to nose it open in order to retrieve food. The placement of inserts into all five incorrect tunnels not only restricted the animal's access to food, but also allowed accurate detection of errors (i.e., attempts to enter an incorrect tunnel) without rewarding such behavior.

Beginning with the third session, the spacing of tunnels along the enclosure wall was randomized prior to each session according to a random number table. In this manner, values were established for the location of the center of each tunnel along the $1.5-\mathrm{m}$ wall. This produced considerable variability in the location of each tunnel from session to session, thereby minimizing the importance of spatial cues. For example, during a randomly selected 1 -month period, the mean location of the center of Tunnel 1 was $25.5 \mathrm{~cm}$ from the start of the 1.5 -m enclosure wall. During this period, the location of Tunnel 1 ranged between 7.5 and $79 \mathrm{~cm}$ along this wall. Comparable data (average location and range) for the remaining five tunnels were as follows: Tunnel 2 : mean $=43 \mathrm{~cm}$, range 20.5-91.5 cm; Tunnel 3: mean $=65 \mathrm{~cm}$, range $33-104 \mathrm{~cm}$; Tunnel 4: mean $=88 \mathrm{~cm}$, range $45.7-117 \mathrm{~cm}$; Tunnel $5:$ mean $=110 \mathrm{~cm}$, range $66-129.5 \mathrm{~cm}$; Tunnel $6:$ mean $=128.5 \mathrm{~cm}$, range $78.8-142.5 \mathrm{~cm}$. Thus, for example, during the 1-month period discussed above, were an animal to leave the startbox, move $79 \mathrm{~cm}$ down the enclosure wall, and enter the nearest tunnel, this strategy might result in entrance into any one of the six tunnels. In short, random variation of tunnel locations minimized the role of spatial cues in reliable selection of the correct tunnel.

When tunnel entry performance had begun to stabilize, training to "count trials" was begun. When the animal returned to the startbox and made physical contact with the guillotine door following Trials 1 and 2, the door was raised to admit the animal. The subject was then manually reinforced with one seed or Noyes pellet. Following completion of Trial 3, the animal was required to cross the enclosure and jump onto the $9 \times 9 \times 46 \mathrm{~cm}$ block of wood, which was centered along the enclosure wall opposite the six tunnels. From the wood block, the rat was able to climb to the top of the enclosure wall $(32 \mathrm{~cm})$ and onto the seat of the office chair (see Figure 1). Finally, the animal was required to climb into its home cage, which was located on the seat of the chair. Following completion of this sequence, the animal was manually rewarded with one seed or Noyes pellet. This signified the end of a session. All sessions were videotaped and scored by two observers. Interobserver reliability approximated 0.9 .

The establishment of both sequences of post-tunnel-entry behavior required some degree of shaping. On those occasions on which the animal made contact with the startbox door on its own following Trial 1 or 2 , it was immediately rewarded with petting and food. However, it was often necessary to manually nudge the animal in the direction of the startbox door, using food (the same as that used to bait the tunnels) to entice it to proceed in the desired direction.

The presence of the wood block in the enclosure occasioned a high enough level of climbing in all 4 successful subjects to allow shaping of the home cage sequence following Trial 3. Food was again used to entice the animal and to reward approximations of the desired sequence. Once both behaviors were established in the animals' repertoires, each subject was cued during early training sessions as to which post-tunnel- 
entry sequence was appropriate. When an error was made during this training period (e.g., contact with the wood block following Trial 1), the experimenter would say "No!" loudly, and push the rat toward the correct area of the cage. Cuing was gradually eliminated and data collection was begun following a maximum of $\mathbf{3 9}$ training sessions. Only data from subsequent sessions, in which cuing was totally eliminated, were analyzed. Tunnel errors were recorded if the animal made initial contact with any but the target tunnel. As in our previous study using this procedure (Davis \& Bradford, in press), the evaluation of tunnelentry data was confined to the initial trial in each three-trial session. Performance on Trials 2 and 3 was excluded from analysis because accuracy, which approached $100 \%$, was most likely due to nonnumerical factors.

"Counting trial" errors were recorded if the animal made physical contact with the wood block following Trial 1 or 2 or if it made contact with the startbox door following Trial 3. Although selection of the target tunnel continued to be reinforced by food, it was not necessary for the subject to select the correct tunnel in order to receive reinforcement for correctly returning to the startbox or home cage. Thus, tunnel selections were evaluated independently of "counting trials."

Intertrial intervals of variable durations were employed in order to control for temporal regularities that might serve as a basis for discrimination. For example, during the final 18 sessions, performance time from the start of the session to tunnel entry on Trial 3 varied between 31 and $291 \mathrm{sec}$.

\section{RESULTS}

Subjects learned not only to enter the correct tunnel based upon its ordinal position, but also to simultaneously "count trials" and emit the appropriate sequence of behavior following each tunnel entry in the session.

A formal criterion was not used to evaluate performance. Rather, subjects were run for between 75 sessions (three blocks) and 175 sessions (seven blocks) so that we could observe the stability of performance over time. As in our previous study (Davis \& Bradford, in press), performance was evaluated using a binomial expansion based upon the number of possible outcomes on each trial. In the case of tunnel entries, performance was nominally

Table 1

Number of Sessions (per Blocks of 25) in Which Subjects Emitted a Correct Sequence of Post-Tunnel-Entry Behaviors

Subject

\begin{tabular}{ccccc}
\hline & \multicolumn{4}{c}{ Subject } \\
& $\begin{array}{c}\text { A } \\
\text { (Tunnel 3) } \\
12 / 25\end{array}$ & $\begin{array}{c}\text { B } \\
\text { (Tunnel 3) } \\
16 / 25\end{array}$ & $\begin{array}{c}\text { C } \\
\text { (Tunnel 4) } \\
17 / 25\end{array}$ & $\begin{array}{c}\text { D } \\
\text { (Tunnel 4) } \\
17 / 25\end{array}$ \\
Session Block & $p<.001$ & $p<.00001$ & $p<.00001$ & $p<.00001$ \\
\hline I & 4 & 4 & 2 & 10 \\
II & 13 & 7 & 2 & 8 \\
III & $9 *$ & 6 & 4 & 7 \\
IV & & 7 & 6 & $9^{*}$ \\
V & & 3 & 5 & \\
VI & & 8 & 7 & \\
VII & & $9 *$ & $7 \dagger$ & \\
\hline
\end{tabular}

Note-The appearance of a correct startbox-startbox-home cage sequence reflected the subject's keeping track of the cumulative number of trials in the session. In addition to counting trials, subjects were required to simultaneously enter the correct tunnel based solely upon its ordinal position in an array. Data were evaluated using $p$ levels derived from a binomial expansion (see text for explanation). Each subject is identified, along with the ordinal position of its target tunnel and the number of correct tunnel entries (with associated $p$ value) recorded during initial trials of the final block of 25 sessions. ${ }^{*} p<.002 . \quad \dagger p=.02$. evaluated with $p=1 / 6$. In every case, tunnel selection was significant by this criterion, as well as by a more conservative $p=1 / 4$ value. Both the rate and asymptotic values of tunnel-entry data were similar to those previously reported (Davis \& Bradford, in press) and will not be discussed beyond summary in Table 1 .

The evaluation of "counting trials" data was based upon a binomial expansion with the probability of a successful (startbox-startbox-home cage) sequence equal to $(1 / 2)^{3}$ or $p=1 / 8$. These results, along with their associated probability levels, are reported in Table 1 . Subjects learned to count trials at different rates. Only Subject $D$ reached and maintained a statistically significant level of performance $(p<.05)$ during the first block of 25 sessions. There was no systematic improvement or deterioration in this subject's performance over 75 additional sessions (see Table 1). The performance of two additional subjects ( $A$ and $B$ ) reached significance during the second block of sessions, and a final subject (C) attained success at the .05 probability level during the fourth block of testing, that is, after 100 sessions. There was little subsequent improvement in subject C's performance over 75 additional sessions.

Errors related to "counting trials" typically consisted of returning to the home cage prematurely, that is, following Trial 1 or 2 . It is worth noting that the simultaneous discrimination procedure involved, in the case of Subjects $C$ and $D$, different numerical requirements (i.e., Tunnel 4 and Trial 3), although there was no indication that this ostensibly more difficult discrimination yielded slower acquisition.

\section{DISCUSSION}

We have demonstrated that rats can learn to enter the correct tunnel based solely upon its ordinal position in an array, while simultaneously keeping track of the cumulative occurrence of three trials in the session. It is important to stress that our subjects' performance did not reveal a high degree of mastery in terms of the absolute number of successful trials. However, their performance was evaluated in terms of a binomial probability that took into account the fact that success consisted not of a single response, but of a sequence of three correct behaviors, each of which occurred in a two-choice situation.

With regard to using the cumulative number of events in a session as a discriminative cue for performance, we have previously demonstrated that the random occurrence of the third shock in a 30-min session could be a salient cue for safety, that is, no further shock (Davis \& Memmott, 1983; Davis, Memmott, \& Hurwitz, 1975). However, the fact that animals can keep track of the number of shock deliveries during a session is less surprising, intuitively, than the present demonstration. There is an obvious difference between enumerating a discrete hedonic event, such as shock, and keeping count of the number of trials run during a session, where duration of the to-be-counted event may be longer and its boundaries less clear.

Our data support the finding by Zorina (1982) that the number of trials may be a sufficient cue for differential performance. However, the present demonstration was accomplished using variable trial durations, a necessary control because temporal regularities in Zorina's procedure may have contributed to her animal's discrimination.

Perhaps of greatest interest is the fact that the present numerical discriminations were accomplished simultaneously. Multiple numerical discriminations have only limited precedent in the literature. Koehler (1950) trained birds to eat several different fixed numbers of food baits de- 
pending upon which color cue was presented. However, his animals performed only one such numerical discrimination at a time, whereas performance in the present experiment involved the simultaneous enumeration of two sets of items during the same session.

\section{REFERENCES}

DAVIS, H., \& BRADFORD, S. (in press). Counting behavior by rats in a simulated natural environment. Ethology.

DAvis, H., \& MrмmotT, J. (1982). Counting behavior in animals: A critical evaluation. Psychological Bulletin, 92, 547-571.

Davis, H., \& MemmotT, J. (1983). Autocontingencies: Rats count to three to predict safety from shock. Animal Learning \& Behavior, 11, 95-100.
Davis, H., Memmott, J., \& HuRWITz, H. M. B. (1975). Autocontingencies: A model for subtle behavioral control. Journal of Experimental Psychology: General, 104, 169-188.

FERSTER, C.B. (1964). Arithmetic behavior in chimpanzees. Scientific American, 210, 98-106.

KOEHLER, O. (1950). The ability of birds to "count." Bulletin of Animal Behavior, 9, 41-45.

Menzel, E. W., \&UNo, C. (1982). Marmosets (Saguinus fuscicollis): Are learning sets learned? Science, 217, 750-752.

ZoRINA, Z. A. (1982). Reasoning ability and adaptivity of behavior in birds. In V. J. A. Novok \& J. Mlikovsky (Eds.), Evolution and environment (pp. 907-912). Prague: CSAV.

(Manuscript received for publication September 19, 1986.) 